\title{
A Linear Array Antenna of Microstrip Patch Antennas Fed by the Open-End of Coplanar Waveguides
}

\author{
Toshihisa Kamei1, Hiromi Shima1, Syotaro Fukuda', Seishiro Ishii ${ }^{2}$ \\ ${ }^{1}$ Department of Communications Engineering, National Defense Academy, Yokosuka, Japan \\ ${ }^{2}$ Kamakami Architecture Design and Quantity Surveying Office, Yamagata, Japan \\ Email: kamei@nda.ac.jp
}

How to cite this paper: Kamei, T., Shima, H., Fukuda, S. and Ishii, S. (2017) A Linear Array Antenna of Microstrip Patch Antennas Fed by the Open-End of Coplanar Waveguides. Wireless Engineering and Technology, 8, 37-49.

https://doi.org/10.4236/wet.2017.82003

Received: February 7, 2017

Accepted: April 11, 2017

Published: April 14, 2017

Copyright (๑) 2017 by authors and Scientific Research Publishing Inc. This work is licensed under the Creative Commons Attribution International License (CC BY 4.0).

http://creativecommons.org/licenses/by/4.0/

\begin{abstract}
In this study, we constructed a 4-element linear array antenna using four 20 $\mathrm{GHz}$ band microstrip patch antennas with a structure such that the signal is fed to the patch antennas from open-end coplanar waveguides without contact. We investigated factors related to the design of linear array patch antennas. To adjust the maximum radiation direction and reduce return loss, we optimized the spacing between the elements and their shape. With an element spacing of $11.50 \mathrm{~mm}$, patch width of $3.90 \mathrm{~mm}$, and patch length of $4.15 \mathrm{~mm}$, we obtained a resonance frequency of $20.05 \mathrm{GHz}$ and a return loss of -29.59 $\mathrm{dB}$ at the resonance frequency. However, in the case of a 4-element linear array antenna structure, undesired resonances occurred in frequency bands other than the design resonance frequency band of $20 \mathrm{GHz}$. To suppress these undesired resonances and obtaining stable operation at the design frequency, we propose a new structure in which the feed line is loaded with a short stub, and show through computer simulations that the occurrence of undesired resonances can be sufficiently suppressed. Furthermore, we demonstrate the problem of radiation gain reduction caused by introducing a short stub, propose a design method for a new structure in which the feed line has slits between the stubs, and show improvement of the antenna gain by $0.5 \mathrm{dBi}$.
\end{abstract}

\section{Keywords}

Microstrip Patch Antenna, Coplanar Waveguide, Linear Array Antenna, Short Stub, Undesired Resonance Suppression

\section{Introduction}

Wireless communication has come into use in a wide variety of applications in 
recent years. The number of services that use the microwave and millimeterwave bands is also increasing. As a result, communication channels are predicted to become more and more diverse. For example, various channels are needed depending on the type of application and its user needs, for example, in aircraft, drones, ships, vehicles, and terminals, all of which require Ka band earth station technology [1] that is multi-platform capable. Adaptive technology that selects among various channels is considered important. For baseband communication using frequencies below microwaves and for communication by devices using middle frequencies, adaptive technology is realized through software-defined radio. For frequencies within the microwave and millimeter-wave bands, however, the development of new adaptive devices that can modulate the phase, amplitude, and frequency of the signal is required. It is challenging to develop adaptive technology that can adaptively control these parameters and flexibly handle the growing diversity of services. We have focused on liquid crystal materials, in which the permittivity acting on high-frequency signals can be changed through electric fields, and we have developed a liquid crystal-loaded phase shifter [2] [3] [4] that enables phase control of the signal. Applying phase shifter to a high-frequency transmission line and antenna enables adaptive selection from among diverse channels.

To show an example of applying this technology, we focused on the Super Hi-Vision service [5], which uses microwaves in the $21 \mathrm{GHz}$ band and is anticipated to become the next-generation satellite broadcast technology. For this technology to become widespread, it is necessary to realize a receiver antenna that has low cost and high performance. We believe that this can best be achieved by simplifying the structure using a liquid crystal-loaded phase shifter and using an adaptive array antenna that realizes antenna gain using array antenna technology. As a fundamental element for achieving this, we chose to use microstrip patch antennas (MSA), which are light-weight and easy to manufacture and for which it is possible to easily change the polarization characteristics and frequency characteristics by changing the element shape. Previous experiments have confirmed that the main beam tilts by controlling the phase of the feed line through the voltage applied to the liquid crystal when a liquid crystal-loaded phase shifter is connected to the feed line of two MSAs [6]. Furthermore, coaxial-coplanar transitions [7] were developed to reduce the loss in liquid crystal-loaded phase shifters constructed with coplanar waveguides with float electrodes (CPW-FE) [3], but transitions such as connectors are necessary to unify these with the antenna. Therefore, we selected a signal feed method that uses an open-end coplanar waveguide (CPW), which is anticipated to reduce the loss due to the waveguide transition by feeding the signal to the patch antenna without contact [8] [9]. Furthermore, we have investigated the effect of the slots of the open-end CPW-fed MSAs on the antenna properties, and proposed a new design method [10]. Based on these results, we demonstrated that it is necessary to create an array of the open-end CPW-fed MSAs in order to obtain radiation gain that is suitable for user needs. 
In this paper, to solve the problems described above, we propose a new design method for a 4-element linear array antenna that uses open-end CPW-fed MSAs having a structure with a unified feed and transmission line consisting of the liquid crystal-loaded phase shifter and patch antenna. For the design, we focused on the shapes of the radiating elements and the spacing between the elements, performed optimization using CST Studio suit 2006, a software package for simulating electromagnetic fields, and proposed using a new structure in which a new short stub is attached to the feed line as a measure to suppress the multiple undesired resonances that appear as a result of the structure of the feed line.

\section{Linear Array Antenna Using Open-End CPW-Fed MSAs}

\subsection{Structure of Linear Array Antenna}

An example of the structure of a 4-element linear array antenna using open-end $\mathrm{CPW}$-fed MSAs is shown in Figure 1. The radiating face is shown in (a), the feeding face is shown in (b), and a cross-section of the part at the dashed line is shown in (c). The patch width $W$ is defined as the length of the patch in the direction perpendicular to the feed line, and the patch length $L$ is defined as the length of the patch in the direction that is parallel to the feed line. The patches are arranged along a straight line, as shown in Figure 1(a). Slots are placed on the back side of the patches, as shown in Figure 1(b). The length of the slots is $100 \mathrm{~mm}$, and the slots are arranged such that their centers are aligned with the centers of the patches. Considering expansion to an adaptive array antenna, we chose a linear array antenna construction for the antenna constituting the fundamental element. Figure 2 shows a conceptual diagram of an adaptive array antenna composed of 16 elements where 4 basic elements are lined up to tilt the beam, in which the basic elements are the 4-element linear array antennas shown in Figure 1. Here, the 4-element linear array antennas are arranged in parallel, and liquid crystal-loaded phase shifters are connected to the feed lines of each one. The tilt of the maximum radiation direction of the main beam in the $x$-axis direction in Figure 2 can be controlled. For the design of the CPWs, the width of the central conductor is set to $a=1.80 \mathrm{~mm}$, the slit width is set to $b$ $=0.10 \mathrm{~mm}$, and the distance between the centers of neighboring patches is set to the patch pitch $d_{p}$ so that the characteristic impedance becomes approximately $50 \Omega$. The printed circuit board has a dielectric thickness of $h=0.50 \mathrm{~mm}$, permittivity of $\mathcal{E}_{r}=2.60$, and conductor thickness of $r=0.018 \mathrm{~mm}$.

\subsection{Design of Linear Array Antenna Using Open-End CPW-Fed MSAs}

In the adaptive array antenna shown in Figure 2, the purpose is controlling the directivity of the main beam through the phase shifters, so we designed the linear array antenna in this investigation such that the maximum radiation direction faces in the direction ( $z$-axis direction) perpendicular to the face in which the linear array antenna is arranged. In the array antenna, the maximum radiation direction is determined by the phase difference of each element, but because the elements in the linear array antenna are arranged on the same feed line, the 


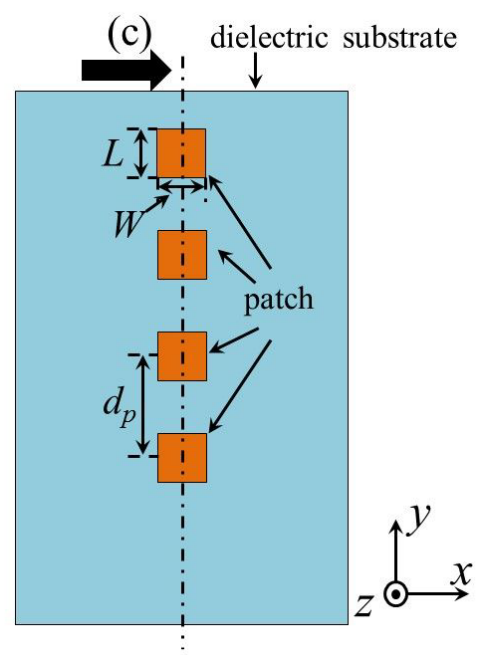

(a)

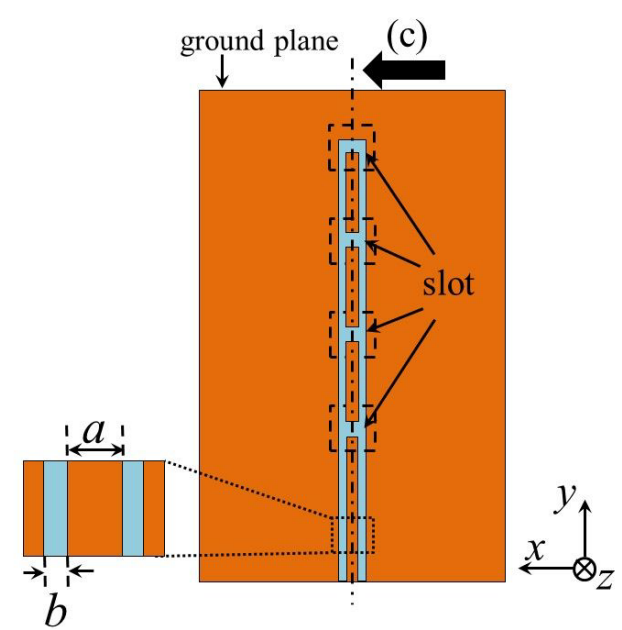

(b)

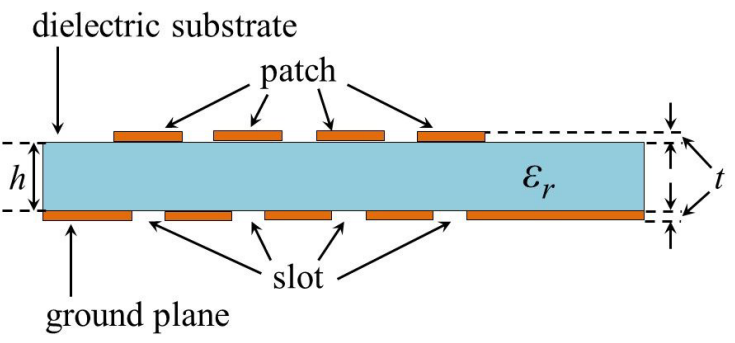

(c)

Figure 1. Structure of linear array antenna with microstrip patch antennas fed by the open end of coplanar waveguides. (a) Radiating face; (b) Feeding face; (c) Cross-section.

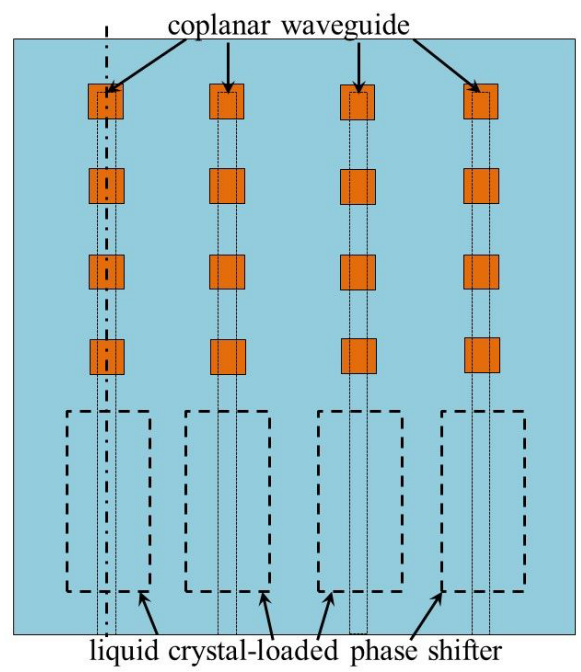

Figure 2. Adaptive array antenna with liquid crystal-loaded phase shifters.

element spacing has a large impact on the relative phase between the elements. The equation for the relationship between the element spacing and the maximum radiation direction in the linear array antenna is shown in Equation (1) [11].

$$
l \sin \theta+\sqrt{\varepsilon_{w}} d_{p}=\lambda_{0}
$$


Here, $\theta$ represents the offset at the $x=0$ face where the $z$-axis direction is taken to be $0^{\circ}$, and $\lambda_{0}$ represents the free space wavelength. The maximum radiation direction is designed to be in the $z$-axis direction, $\theta=0$, so the effective wavelength on the feed line waveguide $\lambda_{g}$ is obtained according to Equation (2).

$$
d_{p}=\frac{\lambda_{0}}{\sqrt{\varepsilon_{w}}}=\lambda_{g}
$$

Based on Equation (2), when making the maximum radiation direction face in the $\mathrm{z}$-axis direction, the element spacing should be set to be equivalent to one wavelength of the radiated electromagnetic wave. Therefore, the element spacing $d_{p}$ was made to vary within the range $11.00-13.50 \mathrm{~mm}$ which corresponds to 0.9 $\lambda_{g}-1.1 \lambda_{g}$, and the electromagnetic field simulation software was used to calculate the changes in the maximum radiation direction. As a result of optimizing the size of the patch in the design of one element [10], we obtained $W=6.90$ $\mathrm{mm}$ and $L=3.90 \mathrm{~mm}$. In Figure 3, an offset of the maximum radiation direction from the $z$-axis to the positive $y$-axis direction is defined to be positive. When the element spacing $d_{p}$ was $11.00 \mathrm{~mm}$, the offset angle became $0^{\circ}$. The radiation characteristics for this situation at $20 \mathrm{GHz}$ are shown in Figure 4, and the frequency characteristics of the reflection loss are shown in Figure 5. Figure 4 shows the radiation characteristics for the $y z$ face, for which the effects of the array are significant. The maximum value of the directional gain was $13.70 \mathrm{dBi}$. In Figure 5, it can be seen that there are several points for which the resonance frequency deviates from the design value of $20 \mathrm{GHz}$. Verifying the electric field distributions at these resonance points, we determined that the resonance point due to phase resonance based on the design value was at $20.45 \mathrm{GHz}$. Therefore, we performed optimization on the patch width $W$ and the patch length $L$ so that this resonance point would become $20 \mathrm{GHz}$. First, to adjust the resonance frequency to $20 \mathrm{GHz}$, we performed an analysis while varying the patch length $L$ in the range $3.90-4.20 \mathrm{~mm}$ at intervals of $0.05 \mathrm{~mm}$. The change in the resonance frequency is shown in Figure 6. The resonance frequency becomes $f_{r}=19.98$ $\mathrm{GHz}$ when $L=4.05 \mathrm{~mm}$. Therefore, we set $L=4.05 \mathrm{~mm}$, and performed an analysis while varying the patch width $W$ in the range $3.50-6.50 \mathrm{~mm}$ at intervals of $0.50 \mathrm{~mm}$ in order to improve the return loss. The change in the reflection loss is shown in Figure 7. The dotted, dash-dotted, and solid lines show the cases in which $W=6.50 \mathrm{~mm}, 5.00 \mathrm{~mm}$, and $4.00 \mathrm{~mm}$, respectively. These results show that as the patch width $W$ becomes smaller, the return loss improves and the resonance frequency increases. This is because the effective wavelength changes along with $W$ because of the change in the effective permittivity directly below the patch. Furthermore, given that the maximum radiation direction changed at the same time due to the change in the patch width $W$, we attempted tore-optimize the element spacing $d_{p}$, patch width $W$, and patch length $L$, and obtained a resonance frequency of $20.05 \mathrm{GHz}$ and return loss of $-29.59 \mathrm{~dB}$ at the resonance frequency when $d_{p}=11.50 \mathrm{~mm}, W=3.90 \mathrm{~mm}$, and $L=4.15 \mathrm{~mm}$. The return loss for this situation is shown in Figure 8, and the frequency characteristics at $20 \mathrm{GHz}$ are shown in Figure 9. Based on the results in Figure 8, it can be 


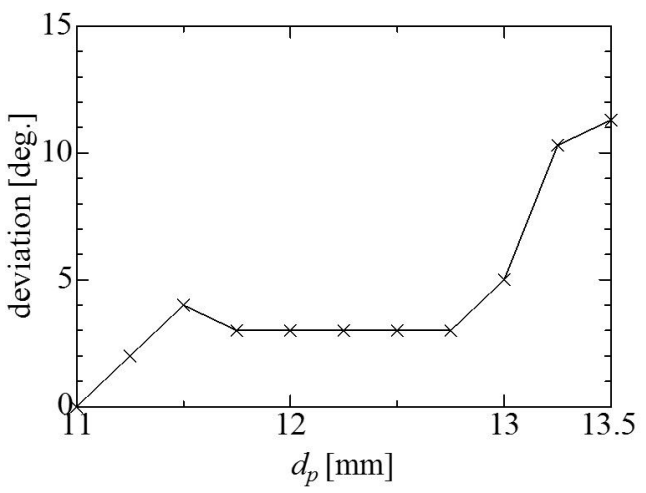

Figure 3. Variation of radiation direction by space between elements.

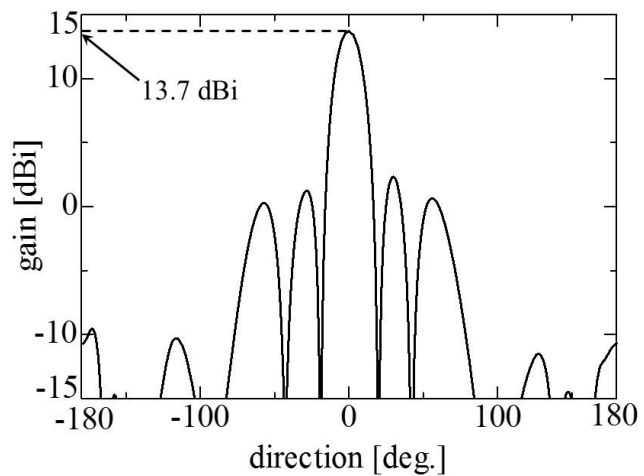

Figure 4. Radiation characteristics of the linear array antenna for $d_{p}=11.00 \mathrm{~mm}$.

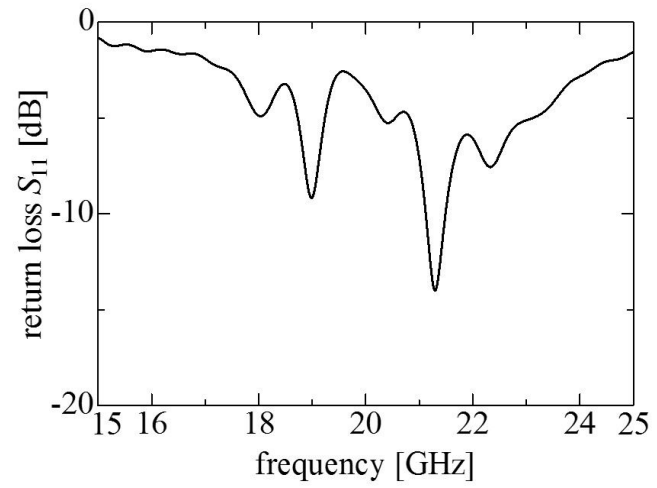

Figure 5. Return loss $S_{11}$ of the linear array antenna for $d_{p}=11.00 \mathrm{~mm}$.

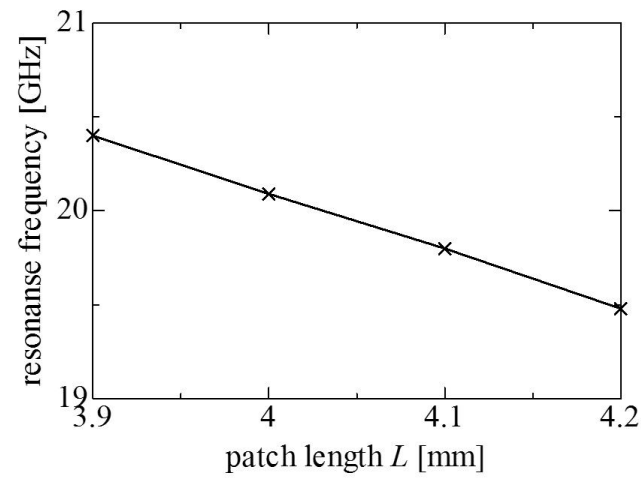

Figure 6. Variation of resonance frequency by patch length. 


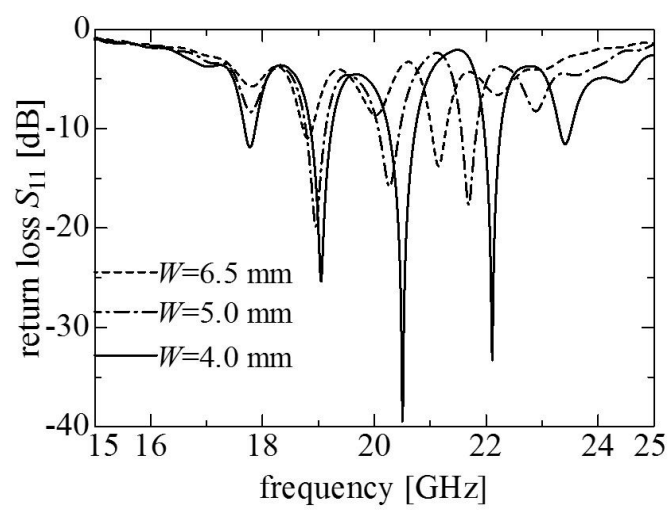

Figure 7. Variation of return loss for patch width $W$.

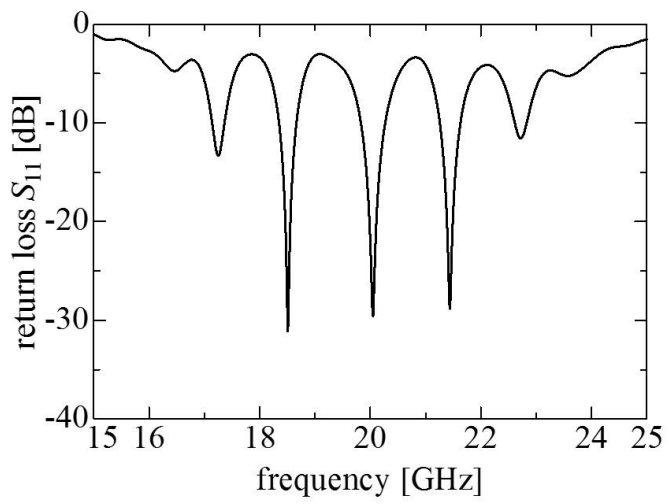

Figure 8. Return loss of the optimal design.

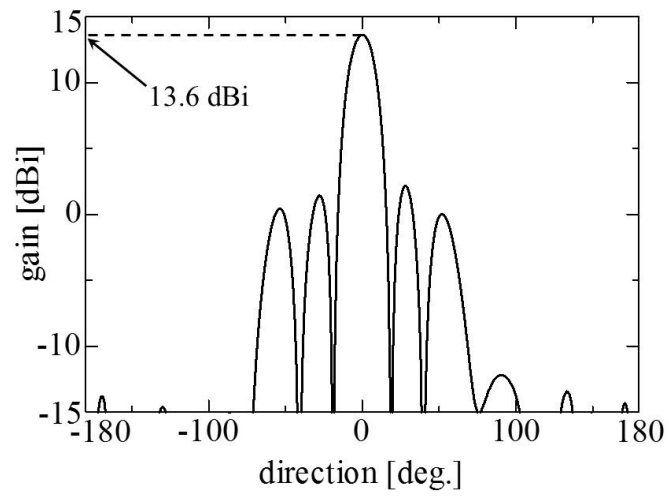

Figure 9. Radiation characteristics of the optimal design.

seen that resonance points exist at frequencies other than $20 \mathrm{GHz}$. The results in Figure 9 show that the maximum value of the directional gain is $13.6 \mathrm{dBi}$, and the maximum radiation direction is the $z$-axis direction. The radiation characteristics at the other resonance point, $18.51 \mathrm{GHz}$, are shown in Figure 10. The maximum radiation direction has changed. Since the radiation direction changes significantly depending on the frequency, it is necessary to suppress undesired resonance points in order to prevent receiving undesired signals. In the next section, we describe a method that uses impedance-matching circuits as a method to suppress undesired resonance points. 


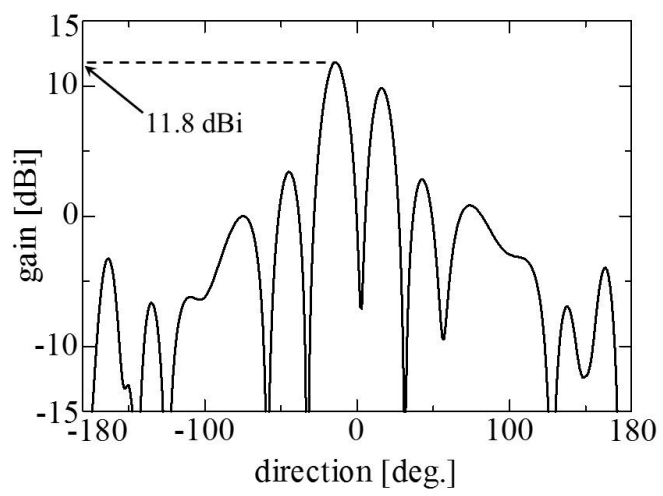

Figure 10. Radiation characteristics for undesirable resonance frequency.

\section{Structure of Stub-Loaded Linear Array Antenna}

\subsection{Investigation of Impedance-Matching Circuit (Stub)}

The 4-element linear antenna considered in the previous section had multiple resonance points. It was demonstrated that the maximum radiation direction changes for resonance points other than $20 \mathrm{GHz}$. In this section, we discuss the results of our investigation on placing an impedance-matching circuit on the $\mathrm{CPW}$ as a method for solving this problem. Previous studies have reported the creation of filters using short stubs for CPWs [12]. According to reference [12], the impedance $Z_{l}$ of the load side separated from the transmission line of line length $I$ can be represented by Equation (3).

$$
Z_{l}=Z_{0} \frac{Z_{l}+j Z_{0} \tan \frac{2 \pi}{\lambda_{g}} l}{Z_{0}+j Z_{l} \tan \frac{2 \pi}{\lambda_{g}} l}
$$

To match impedances, the reactance of the parallel stub is represented as $j_{X}$, and the designer should connect a circuit with $j_{X}$ needed to cancel the imaginary part of $Z_{l}$ shown in Equation (3). Here, the parameters of the stub that can be adjusted include the line length $l$ and stub reactance $j_{X}$. Considering the impedance of the stub side as viewed from the feed line, the short stub represents the case where $Z_{l}=0$ in Equation (3), so it can be expressed as shown in Equation (4).

$$
Z_{l}^{\prime}=j Z_{0} \tan \frac{2 \pi}{\lambda_{g}} l
$$

In the 4-element linear array antenna considered here, it is necessary to adjust the impedance-matching circuit to suppress the resonances at frequencies other than the design frequency of $20 \mathrm{GHz}$. In other words, setting the line length $I$ to $\lambda_{g} / 2$ so that $Z_{l}$ in Equation (4) becomes zero changes the impedance at wavelengths other than $\lambda_{g}$, making it is possible to suppress the undesired resonance points at frequencies other than $20 \mathrm{GHz}$.

\subsection{Design of the Stub Circuit}

The stub circuit used to load the feed line on the back face of the linear array an- 
tenna under consideration is shown in Figure 11. A short stub with a shortened tip is used. The stub length is defined as $L_{s}$, and the distance from the feed point to the stub is defined as $d_{c}$. The central conductor width and slit width of the stub are $a=1.80 \mathrm{~mm}$ and $b=0.10 \mathrm{~mm}$, similar to the feed line. The adjustable parameters of the stub include the stub length and the stub arrangement positions. In this investigation, we set the stub length $L_{s}$ to $\lambda_{g} / 2=6.10 \mathrm{~mm}$, and analyzed the changes in the antenna characteristics with respect to changes in dc using the electromagnetic field simulation software. The return loss $S_{11}$ at 20 $\mathrm{GHz}$ is shown in Figure 12. The largest return loss of $-25.22 \mathrm{~dB}$ was obtained when $d_{c}=37.50 \mathrm{~mm}$. Next, we analyzed the changes in the characteristics with respect to changes in the stub length $L_{s}$. The changes in return loss at $20 \mathrm{GHz}$ are shown in Figure 13. Because we obtained $S_{11}=-46.5 \mathrm{~dB}$ when the stub length was $L_{s}=5.95 \mathrm{~mm}$, we determined that this is the optimal value. Furthermore, the frequency characteristics of the return loss for this situation are shown in Figure 14, and the radiation characteristics are shown in Figure 15. The solid line shows the return loss frequency characteristics for the case with the stub, and the dotted line shows the characteristics for the case without the stub. Figure 14 shows that the undesired resonances are suppressed well, and that the return loss at the design frequency has been improved. On the other hand, as shown in Figure 15, the gain in the maximum radiation direction decreased.

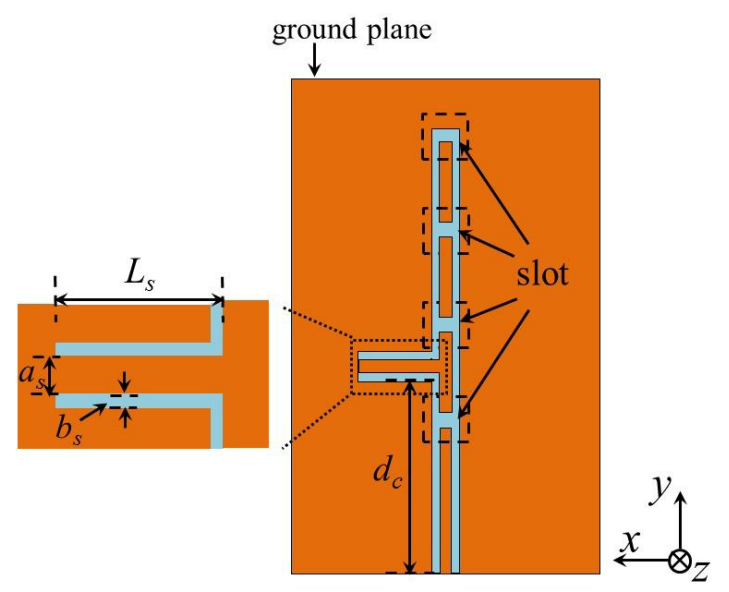

Figure 11. Structure of the stub circuit.

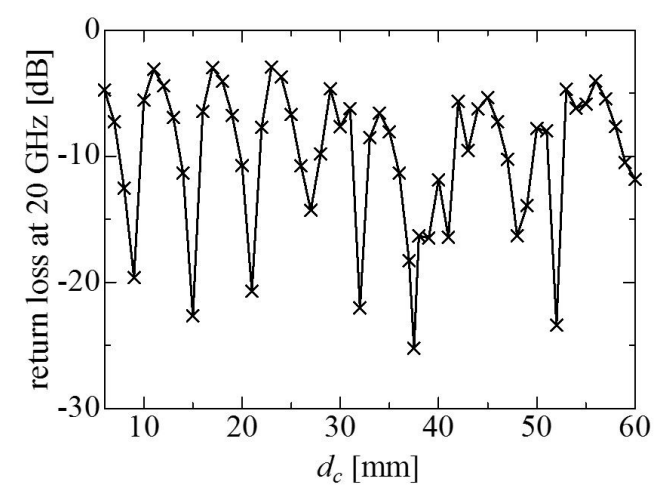

Figure 12. Variation of return loss by distance between the stub and feeding point $d_{c}$. 


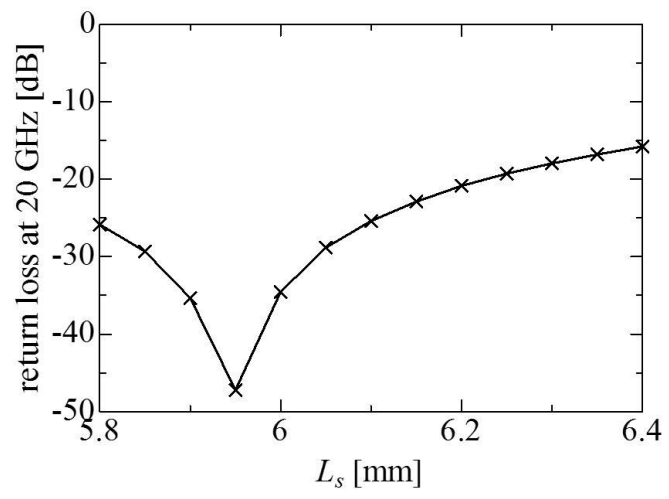

Figure 13. Variation of return loss by stub length $L_{S}$.

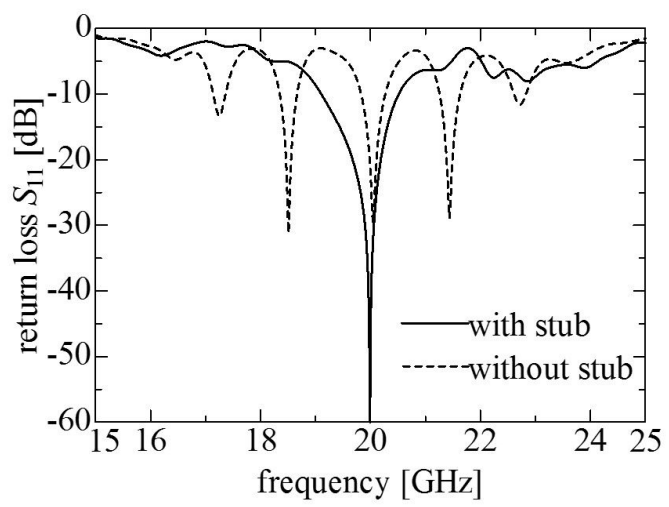

Figure 14. Return loss of the linear array antenna with stub.

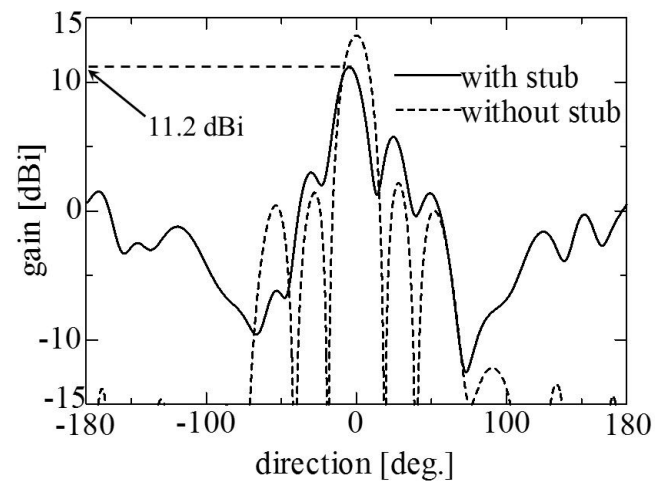

Figure 15. Radiation characteristics of linear array antenna with stub.

While the gain was $13.6 \mathrm{dBi}$ without the stub, the gain was $11.2 \mathrm{dBi}$ with the stub. This is believed to be caused by the stub radiating waves from the face with the feed line at the back of the antenna. In addition, the side lobes increased.

\subsection{Design of Stub Circuit with Slits}

In the previous section, we demonstrated that loading with the stub circuit was effective for suppressing undesired resonances, but the gain between the patch face and the opposite face with the feed line increased due to radiation from the stub. Therefore, we attached a stub circuit with a slit as shown in Figure 16. We propose a method for reducing the backward radiation by strengthening the 
coupling between the stubs. This is done by generating aperture coupling between the stubs by creating slits within the stubs. Since the value of $\lambda_{g}$ in the stub circuits may change due to the slits, we performed optimization of the stub length $L_{s}$. Figure 17 shows the changes in the return loss with respect to the stub length $L_{s}$. When $L_{s}=5.70 \mathrm{~mm}, S_{11}=-42.53 \mathrm{~dB}$, and $f_{r}=19.99 \mathrm{GHz}$. This was chosen as the optimal value. The return loss for this situation is shown in Figure 18, and the radiation characteristics are shown in Figure 19. For return loss, the

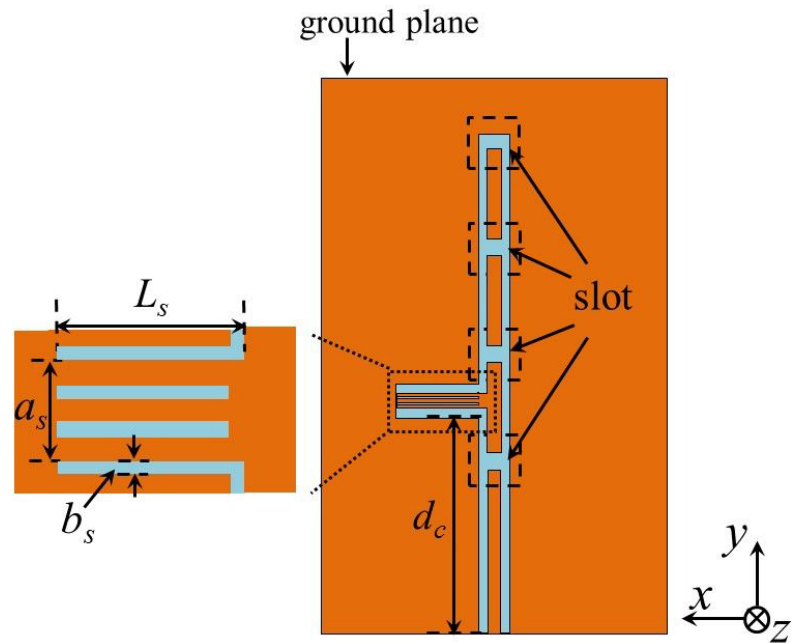

Figure 16. Structure of stub circuit with slits.

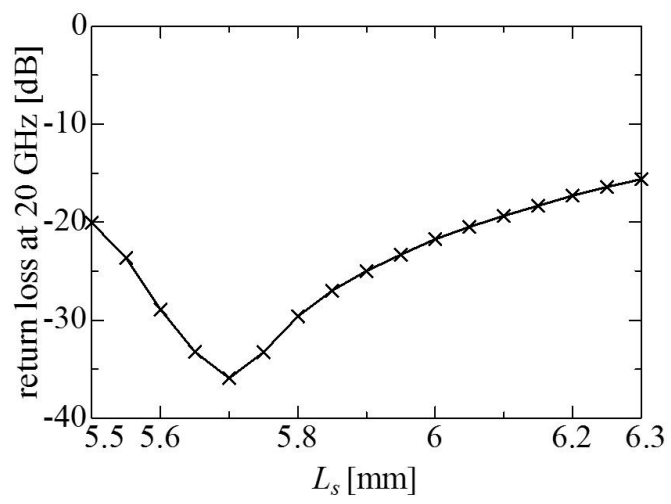

Figure 17. Variation of return loss by stub length $L_{S}$.

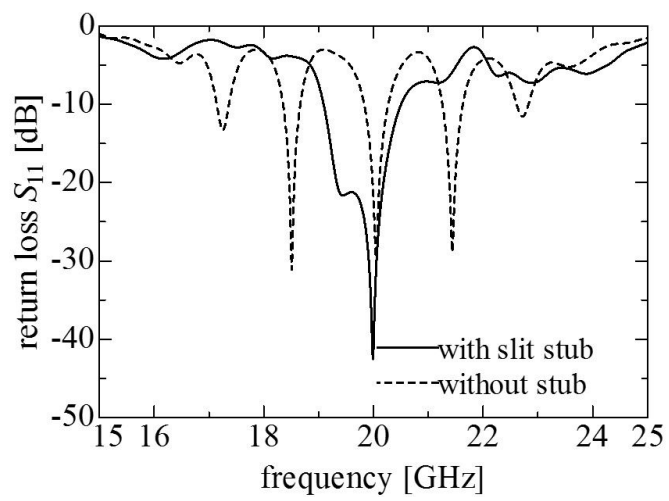

Figure 18. Return loss of the linear array antenna with slit stub. 


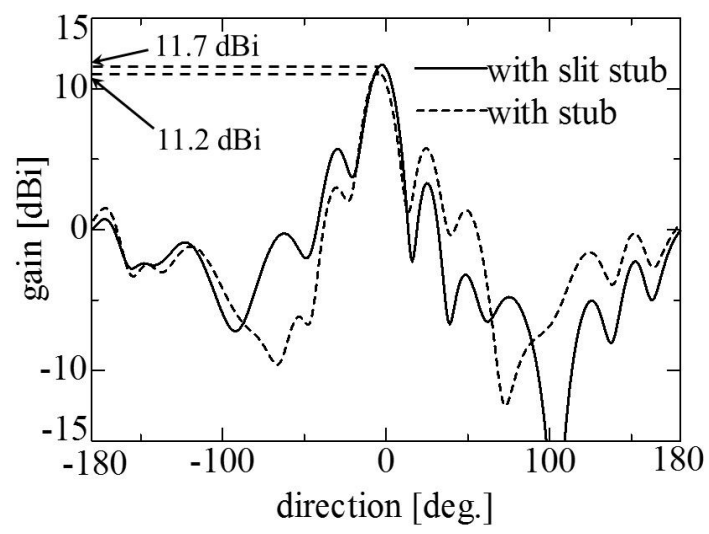

Figure 19. Characteristics of te linear array antenna with slit stub.

undesired resonances were suppressed after loading with the stub as well, and no large changes were seen. For radiation characteristics, on the other hand, a gain improvement of $0.5 \mathrm{dBi}$ was seen.

\section{Conclusion}

We investigated the design of a linear array patch antenna composed of 4-element linear array antennas using four $20 \mathrm{GHz}$ band MSAs with a structure such that the signal is fed to the patch antennas from open-end CPWs without contact. To adjust the maximum radiation direction and reduce the return loss, we optimized the element spacing and the element shape, and obtained a return loss of $-29.59 \mathrm{~dB}$ at the resonance frequency of $20.05 \mathrm{GHz}$. However, undesired resonance frequencies gave rise to frequency bands other than the design frequency of $20 \mathrm{GHz}$ when a 4-element linear array antenna structure was used. To suppress these undesired resonances, we proposed a new structure in which the feed line is loaded with a short stub as a method to obtain stable performance at the design frequency. We demonstrated that this method can sufficiently suppress the undesired resonances. Furthermore, we revealed that introducing the short stub causes a new problem in which the radiation gain is reduced. To solve this problem, we proposed a design method for a new structure in which the circuit has slits between the stubs, which improved the antenna gain by $0.5 \mathrm{dBi}$.

In the future, we plan to investigate methods for further improving the maximum radiation gain of a linear array of patch antennas that is designed using the design method proposed in this paper.

\section{References}

[1] International Telecommunication Union (2016) Use of the Frequency Bands 19.7 20.2 GHz and 29.5 - 30.0 GHz by Earth Stations in Motion Communicating with Geostationary Space Stations in the Fixed Satellite Service. Resolution 156. Proceedings of the World Radiocommunication Conference (WRC-15), Geneva, 2-27 November 2015.

[2] Kamei, T., Utsumi, Y., Moritake, H., Toda, K. and Suzuki, S. (2003) Measurements of the Dielectric Properties of Nematic Liquid Crystal at $10 \mathrm{kHz}$ to $40 \mathrm{GHz}$ and Application to a Variable Delay Line. Electronics and Communications in Japan (Part 
II: Electronics), 86, 49-60. https://doi.org/10.1002/ecjb.10130

[3] Utsumi, Y., Kamei, T., Saito, K. and Moritake, H. (2005) Increasing the Speed of Microstrip-Line-Type Polymer-Dispersed Liquid-Crystal Loaded Variable Phase Shifter. IEEE Transactions on Microwave Theory and Techniques, 53, 3345-3353. https://doi.org/10.1109/TMTT.2005.857123

[4] Utsumi, Y., Kamei, T., Maeda, T. and Dinh, N.Q. (2007) Microwave High-Speed Liquid Crystal Devices Using CPW with Floating Electrode. Molecular Crystals and Liquid Crystals, 476, 249-259. https://doi.org/10.1080/15421400701739121

[5] International Telecommunication Union (2012) Recommendation, ITU-R BO. 1776-1. Maximum Power Flux-Density for the Broadcasting-Satellite Service in the Band 21.4-22.0 GHz in Regions 1 and 3. International Telecommunication Union, Geneva.

[6] Kamei, T., Yokota, M., Ozaki, R., Moritake, H. and Onodera, N. (2011) Microstrip Array Antenna with Liquid Crystals Loaded Phase Shifter. Molecular Crystals and Liquid Crystals, 542, 689-697. https://doi.org/10.1080/15421406.2011.570555

[7] Kamei, T., Utsumi, Y., Dinh, N.Q. and Thanh, N. (2007) Wide-Band Coaxial-toCoplanar Transition. IEICE Transactions on Electronics, E90-C, 2030-2036. https://doi.org/10.1093/ietele/e90-c.10.2030

[8] Kamei, T., Ohshima, Y. and Kawano, T. (2014) A Study for Patch Length L of Microstrip Patch Antenna Excited By Coplanar Waveguide Edge Slot. Proceedings of APCOM 2014, Tokyo, 24-27 August 2014, 76-79.

[9] Ohshima, Y., Kamei, T. and Kawano, T. (2014) Design of Microstrip Patch Antenna Excited by Coplanar Waveguide Edge Slot. Proceedings of Asia-Pacific Microwave Conference 2014, Sendai, 4-7 November 2014, FR3G-39, 1396-1398.

[10] Ohshima, Y., Kamei, T. and Kawano,T. (2015) A Study for Design of Microstrip Patch Antenna Fed by Open-End of Coplanar Waveguide. IEICE Transactions on Electronics, J98-C, 348-355.

[11] Lalezari, F. and Massey, C.D. (1987) MM-Wave Microstrip Antennas. Microwave Journal, 30, 87-96.

[12] Takiguchi, Y., Ma, Z. and Kobayashi, Y. (2000) SCE2000-26/MW2000-90 Design of a Novel $30 \mathrm{GHz}$ Band-Pass Filter Using Coplanar Waveguide Structures [in Japanese]. Technical Report of IEICE, 100, 105-109.

\section{Submit or recommend next manuscript to SCIRP and we will provide best service for you:}

Accepting pre-submission inquiries through Email, Facebook, LinkedIn, Twitter, etc. A wide selection of journals (inclusive of 9 subjects, more than 200 journals)

Providing 24-hour high-quality service

User-friendly online submission system

Fair and swift peer-review system

Efficient typesetting and proofreading procedure

Display of the result of downloads and visits, as well as the number of cited articles

Maximum dissemination of your research work

Submit your manuscript at: http://papersubmission.scirp.org/

Orcontactwet@scirp.org 